\title{
Multi-Element Fiber for Space-Division Multiplexed Optical Communication system
}

\author{
S. Jain*, V. J. F. Rancaño, T. C. May-Smith, P. Petropoulos, J. K. Sahu, \\ and D. J. Richardson \\ Optoelectronics Research Centre, Southampton, SO17 1BJ, U. K. \\ *sj3g11@orc.soton.ac.uk
}

\begin{abstract}
This paper reviews a novel approach of implementing space-division multiplexing (SDM) based on Multielement Fiber (MEF) technology. The MEF geometry provides for ultra-low crosstalk between the spatial channels while full compatibility with standard WDM components is maintained. Passive Ge-doped 3-MEFs have been fabricated and characterized and error free WDM data transfer at an aggregate rate of 1014 Gbit/s shown. Also, an Er/Yb-doped cladding-pumped 5-MEF has been demonstrated which enables pump sharing between the signal fiber-elements thereby reducing the number of components in the system. Both these passive and active MEFs have been used to demonstrate an error free SDM system with in-line amplification.
\end{abstract}

Keywords: Space-division multiplexing, Optical fiber communication, Optical fiber amplifier

\section{INTRODUCTION}

Since the deployment of standard single mode fibers (SSMFs), there have been numerous developments to increase the bandwidth up to the 100Tbit/s levels demonstrated in the latest research. The research in SSMF is now approaching the fundamental capacity limit, and the spatial dimension seems to be the only approach that can substantially increase the per-fiber data capacity [1]. The most intuitive solution to cope with the foreseeable capacity crunch is to deploy a greater number of SSMF systems. However, that would entail huge economic cost leading to diminishing returns that would handicap the growth of future communication networks.

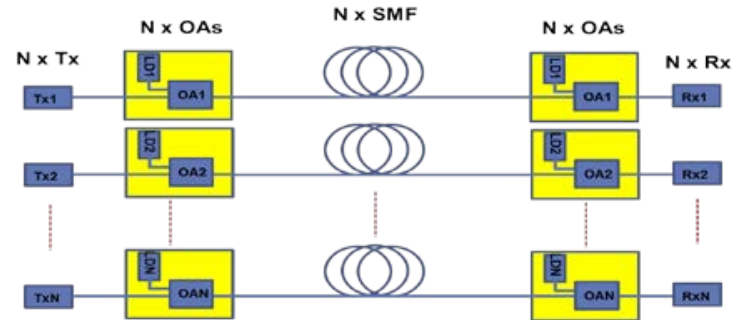

a)

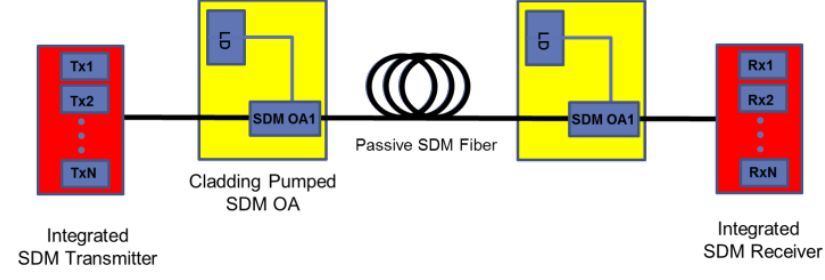

b)

Fig1. Schematic of a network showing a) N-parallel WDM systems, and b) SDM system

In recent years, space-division multiplexing (SDM) has emerged as a solution to scale up the capacity beyond WDM non-linear limit with favourable economics. Implementation of SDM in optical fiber is expected to reduce the overall cost required for capacity scaling. Moreover, it would be a longer term solution as compared to deployment of SSMF systems in parallel. Multicore fiber (MCF) and multimode fiber (MMF) have been proposed as the two main approaches for implementing SDM in optical fibers. As shown in Fig.1, these fibers allow for integration of the transceiver, amplification and multiplexing components relative to SMF system. Both of these technologies have shown impressive results in a very short time span [2]. Multiplexing of up to 19 cores have been demonstration in MCF, whereas passive few mode fiber (MMF) supporting 6 spatial modes have also been demonstrated [3-4].

However, there are inherent limitations in scaling the capacity through these technologies which prevents SDM being exploited to its full potential. The number of cores that can be incorporated in a fiber is currently limited due to the restriction on overall cladding diameter due to mechanical reliability consideration and minimum core-to-core pitch to enable low crosstalk operation [5]. In MMF, multiple-input-multiple-output (MIMO) signal processing schemes are necessary to accommodate mode coupling, which decreases the network flexibility. Special multiplexers (MUX) are also required and have been demonstrated for both MCF [6] and MMF [7], but ensuring compatibility with off-the-shelf WDM components requires modification of at least some of them. Techniques that provide for low cross-talk, that offer potential for greater scalability whilst still offering significant device integration opportunities are thus of great current interest. 


\section{Multi-Element Fiber}

Multi-element fiber (MEF) has been demonstrated as an alternative technology to implement SDM in optical fibers that can avoid some of the challenges faced in MCF and MMF approaches. MEF consists of multiple fiber-elements that are coated together in a common coating during fiber fabrication. MEF technology can be used to fabricate both SDM transmission fibers as well as amplifiers depending on the MEF preform assembly. Moreover, it is compatible with both MCF and MMF, and even has the potential to be implemented in conjunction with MCF and MMF. The fiber-elements in an MEF behave as independent fibers in-isolation, and exhibit ultralow crosstalk levels. Moreover, the fiber-elements in MEF can be easily accessed simply by stripping the polymer coating, and conventional splicing can be used for interconnection thereby avoiding the need for developments of SDM couplers. This also enables the MEF to be backward compatible with SSMFs providing the required flexibility in the network and inter-operability with existing systems.

MEF technology was first demonstrated for core-pumped SDM amplifiers [8]. A crosstalk free 3-element MEF (3-MEF) and 7-element MEF (7-MEF) Er-amplifier was demonstrated. The cross-sectional image of our 7-MEF core-pumped amplifier fiber is shown in Fig. 2a. However, this approach does not fully exploit the cost saving that can be achieved through MEF technology due to the requirement of using one (single-mode) pump diode per core. To address this, a cladding-pump implementation was developed which uses a single shared pump diode, greatly improving the cost efficiency of the scheme (see fig. $2 \mathrm{~b}$ for cross-section) [9]. The pump is launched into the coreless pump delivery fiber-element which then gradually couples the pump light into signal fiber-elements in MEF through evanescent coupling. Efficient pump transfer to signal fiber-elements requires that optical contact is maintained between the pump and signal fiber-elements. MEF technology has also been extended to fabricate passive SDM fibers for data transmission itself, the cross section images for which are shown in Figs. 2c and 2d [10,11]. A schematic of the assembled preform for a 7-MEF on the draw tower is shown in Fig.2e.

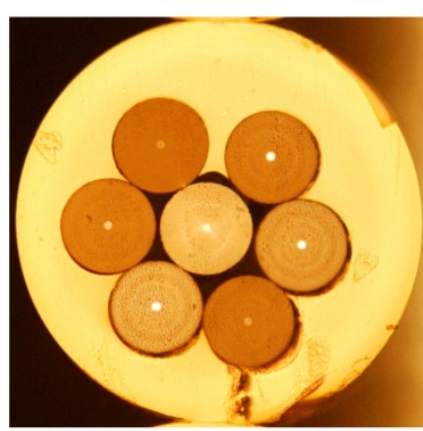

a)

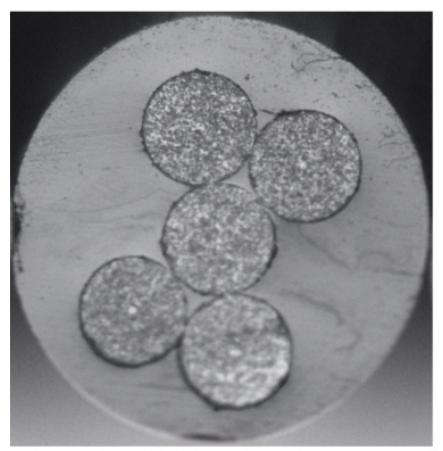

b)

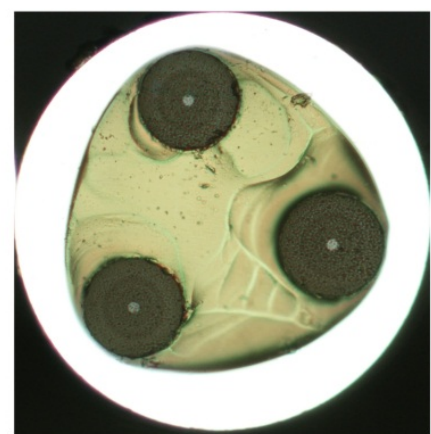

c)

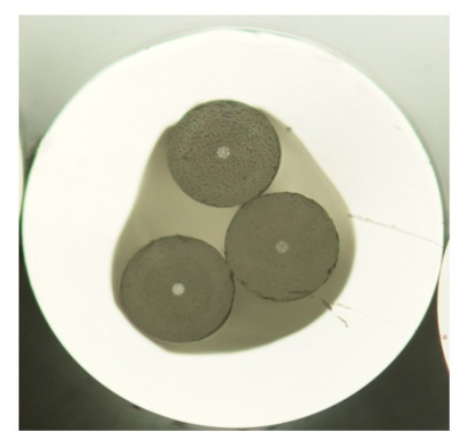

d)

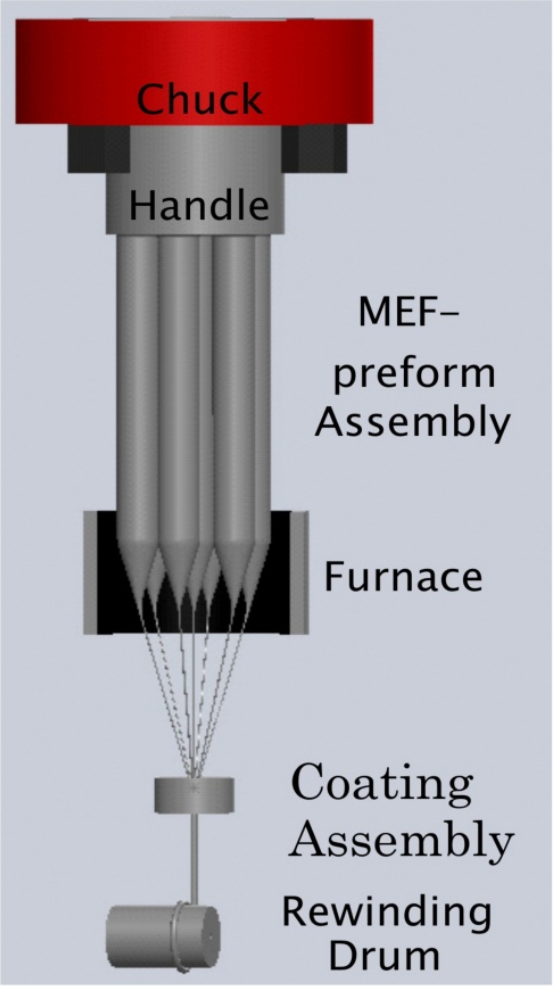

e)

Fig2. Cross-section image of MEFs fabricated for a) core-pump amplifier, b) cladding-pump amplifier, c) and d) transmission fiber, and e) schematic of the 7-MEF preform on the draw tower.

\section{Cladding-Pumped Multi-Element Er/Yb-doped Fiber Amplifier}

A 5 element MEF (5-MEF) comprising 4 Erbium(Er)/Ytterbium(Yb)-doped signal fiber elements surrounding a central coreless pump fiber-element was fabricated [9]. A 4m length of fiber was used to design the amplifier in the C-band. The gain and noise figure (NF) characteristics and pump power vs gain profile of the amplifier is shown in Fig. 3a and 3b respectively. A maximum gain of $34 \pm 2 \mathrm{~dB}$ per fiber-element was obtained in the C-band using a $960 \mathrm{~nm}$ multimode mode pump at $6.4 \mathrm{~W}$ of output power. The core absorption in signal fiber-elements 
varied between $36-61 \mathrm{~dB} / \mathrm{m}$ at $1536 \mathrm{~nm}$ due to different Er concentration in the fiber-elements. This also resulted in the gain variation between the elements. It can be seen from Fig. $2 b$ that the gain saturated for more than $8 \mathrm{~W}$ of launched pump power.

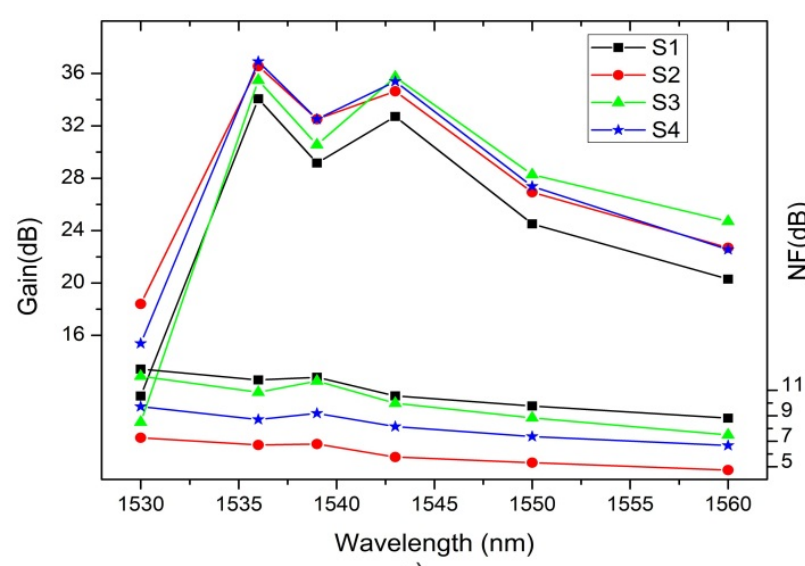

a)

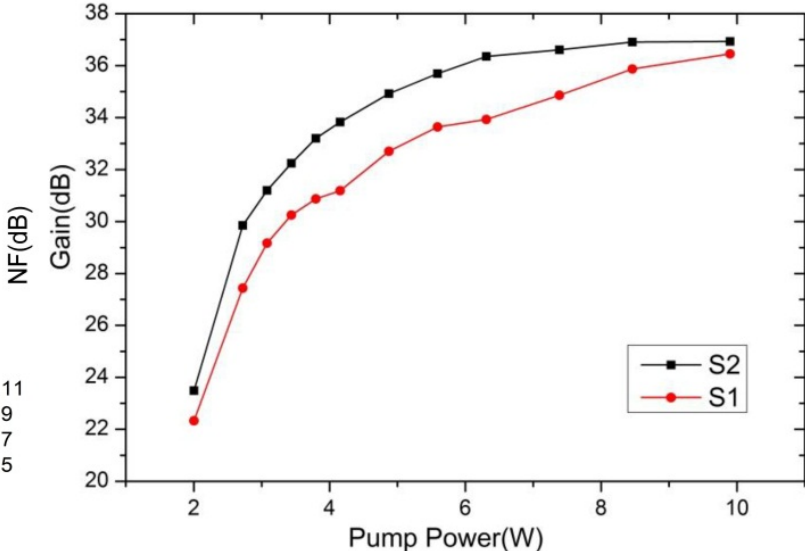

b)

Fig3. a) Gain and NF characteristics of signal fiber-elements (S1, S2, S3, and S4) in cladding-pump amplifier, and b) pump power versus gain spectra for two of the fiber-elements.

The amplifier was also tested in a multichannel configuration to check its WDM performance. The MEF amplifier provides flexibility in changing the length of the amplifier which in turn can be used tune the gain profile. The signal fiber-elements were cascaded one-by-one to tune gain profile of the amplifier. Fig. 4a and 4b show the spectra for 2-cascaded and 3-cascaded configuration which illustrates the gain peak shifts towards longer wavelength as the fiber-elements are cascaded. It can be seen that, as expected, the greater the number of fiber-elements cascaded the greater the gain shifts towards longer wavelength.

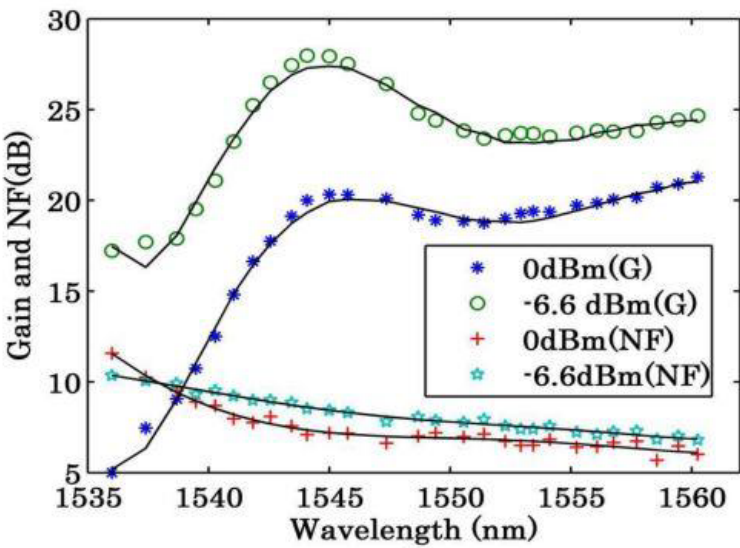

a)

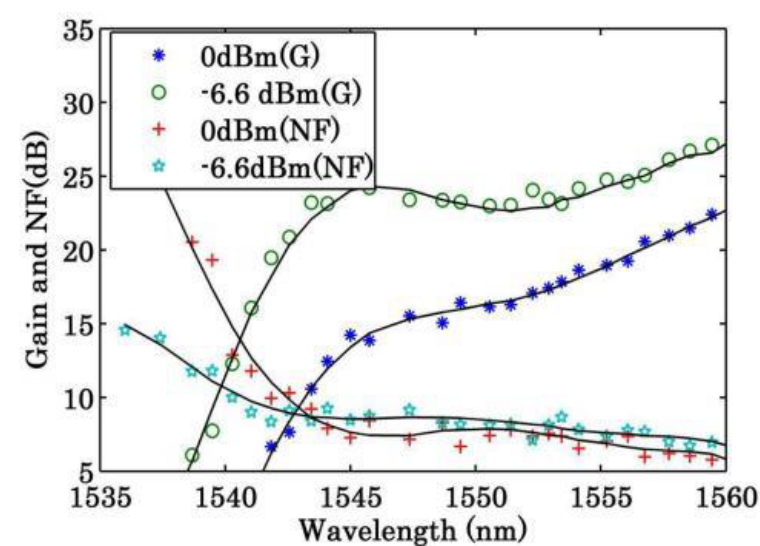

b)

Fig4. WDM characteristics of amplifier for a) 2-cascade, and b) 3-cascade

The gain profiling characteristics have also been exploited to demonstrate a broadband amplifier covering both the $\mathrm{C}$ and $\mathrm{L}$ band using a split-band configuration [12]. In this case, one signal fiber-element was used in the Cband while other signal fiber-elements were cascaded to extend the gain in the L-band. Using this configuration, it was shown that it is possible to develop an amplifier providing $>20 \mathrm{~dB}$ gain in a $>80 \mathrm{~nm}$ bandwidth covering both the $\mathrm{C}$ and $\mathrm{L}$ band. The MEF amplifier provides the flexibility to change the effective length of the amplifier, which has been a rigid constraint before, simply by connecting a different number of signal fiber-elements together. This enables the development of a reconfigurable amplifier, in which the gain profile can be tuned depending on the requirement.

\section{Passive Multi-Element Fiber}

The deployment of optical fibers necessarily involves significant capital expenditure and the mechanical and optical reliability of fibers for telecommunication applications is a critical issue that cannot be avoided. It is required that the fiber must be sufficiently durable to withstand harsh environmental conditions without any decrease in performance. To test mechanical reliability several passive 3-MEFs with different separation were fabricated [10]. The 3-MEFs in which the elements were well separated (see Fig. 1c) showed 400kpsi of proof strength, and the loss was similar to the loss of single fibers drawn from same preform. However, the strength of MEF decreased in cases where the fiber-elements touched other (see Fig. 1d) indicating that some minimum 
separation is required between fiber-elements. There doesn't seem to be any immediate limitation to scaling up the MEF to incorporate more spatial channels if the critical minimum separation between fiber-elements is maintained. It should be noted that this requirement makes the MEF fabrication for telecommunication different from that of cladding-pump amplifiers. This arises from the different usage of both types of fibers. Fiber amplifiers are devices where the fibers are packaged in safe boxes, with less demanding mechanical robustness requirements. Indeed commercial high power fiber laser products based on this technological approach have existed on the market for several years now.

The fabricated MEFs were then tested for BER characteristics using 28-10Gbit/s OOK, 2-40Gbit/s BPSK, and 224Gbit/s QPSK channels with an overall rate of $1014 \mathrm{Gbit} / \mathrm{s}$. All channels were retrieved error-free in all the passive MEFs. Moreover, the MEF provided ultralow crosstalk between spatial channels $(<-80 \mathrm{~dB})$. The low level of crosstalk assured that the fiber-elements behaved as independent fibers.

An amplified SDM system was also demonstrated which consisted of 3-MEFs and cladding-pump amplifier[11]. Standard SMF pigtails were spliced at each end of the fiber-elements of the MEFs, and they were connected using standard components. This demonstrates the backward compatibility with current WDM systems and components provided by MEFs with deployed SSMFs. The cladding-pump MEF amplifier was then used at the end of each line to amplify the transmitted signals. Most channels were retrieved error free after one amplified span. However, due to the ripple in the gain profile error-free performance for channels away from peak gain was not achieved. The gain profiling characteristics of the amplifier were then used to shift the gain in longer wavelength and retrieve the channels [10-11].

\section{Conclusion}

Multi-element fiber technology is proposed as a candidate to implement SDM in optical fibers and amplifiers. The technology meets telecommunication demands for backwards compatible, flexible implementation of SDM in transmission fibers. It provides for ultralow crosstalk between the spatial channels, and their easy access allowing standard splicing to be used. In a cladding-pump configuration, MEF offers benefits through components sharing which can reduce the overall cost of the system.

\section{References}

[1] P. J. Winzer, "Modulation and multiplexing in optical communication systems," LEOS Newsletter, February, 4 (2009).

[2] D. J. Richardson, J. M. Fini, and D. N. Nelson, "Space-divison multiplexing in optical fibers," Nat. Photonics 7(5), 354-362, (2013).

[3] J. Sakaguchi et. al., "19-core MCF transmission system using EDFA with shared core pumping coupled via free-space optics,” Opt. Exp., 22(1), 90 (2014)

[4] Ryf, R. et al., “12 x 12 MIMO Transmission over 130-km Few-Mode Fiber,” Proc. Frontiers in Optics 2012, PDP FW6C.4. (2012)

[5] S. Matuso et. al., "Large-effective area ten-core fiber with cladding diameter of about $200 \mu$ m," Opt. Letters, 36(23), 4626, (2011).

[6] K. Wantabe et. al., "Development of fiber bundle type fan-out for multicore fiber," in OECC, 5C1-2, (2012).

[7] S. G. Leon-Saval et. al., "Photonic lanterns: a study of light propagation in multimode to single-mode converters,” Opt. Express, 18(8), 8430, (2010).

[8] S. Jain et. al., "Er-doped multi-element fiber amplifier for space-division multiplexing operations", in Opt. Letters, 38(4), 582, (2013).

[9] S. Jain et. al., "Cladding-pumped Er/Yb-doped multi-element fiber amplifier," in WSOF, 5.4, (2013).

[10] S. Jain et. al., "Multi-element fiber for space-division multiplexing operations,” Opt. Exp., 22(4), 3787, (2014).

[11] V. J. F. Rancaño et. al., "First demonstration of amplifier transmission line based in multi-element fiber technology,” in ECOC, PD1.C.2, (2013).

[12] S. Jain et. al., "Cladding-pumped multi-element fiber amplifier for C+L band operations,” in OFC, M2J.3, (2014). 